\title{
Congenital anterior urethral diverticulum in male child \\ ${ }^{*}$ Rahman MA
}

\begin{abstract}
Congenital anterior urethral diverticulum is an uncommon condition in males. In my series a 15 years old boy with congenital anterior urethral diverticulum was presented with urinary symptoms and ventral penile swelling. The diagnosis was made on retrograde urethrogram (RGU) and micturiting cystourethrogram (MCU). The patient had normal renal function. Open diverticulectomy and primary urethral reconstruction was carried out uneventfully.
\end{abstract}

CBMJ 2018 January: vol. 07 no. 01 P: 45-47

keywords : Anterior urethral diverticulum, Open diverticulectomy

\section{Introduction}

A urethral diverticulum is a condition where the urethra push into the connective tissue layers (fascia) that surround it. ${ }^{1,2}$ Congenital anterior urethral diverticulum is an uncommon condition in males and classified into saccular variety and globular variety, the former being more common than the latter. ${ }^{3}$ Urethral diverticulum generally located in the ventral aspect of the anterior urethra, though it can be found anywhere from the bulbous to the mid pendulous portion of the urethra. ${ }^{4}$ We describe the case of a 15 years old boy with a congenital anterior urethral diverticulum who presented with ventral penile swelling. The diagnosis was made on RGU and MCU. The patient had normal renal function. Open diverticulectomy and primary urethral reconstruction was carried out uneventfully.

\section{Case Summary}

A 15 year-old male presented with complaints of poor urinary stream, post void dribbling and a swelling on the ventral aspect of penile urethra. The swelling was soft, cystic, fluctuant, free from the overlying skin, nontender and compressible, and it just collapsed completely on digital compression with urine coming out per urethra. Routine urine analysis, urine culture, routine blood counts and serum creatinine were normal. Ultrasonography revealed both kidneys normal in size and shape and with normal urinary bladder. Retrograde and micturating urethrogram was done, and it revealed the presence of a widemouthed saccular diverticulum involving most of the penile urethra and the proximal lip of diverticulum form an acute angle with the normal caliber proximal urethra and normal urinary bladder without any vesicoureteric reflux (Figure-1). However, the average urinary flow rate was only $4.1 \mathrm{~mL} / \mathrm{s}$ and maximum flow rate was $9.2 \mathrm{ml} / \mathrm{s}$. Patient was managed by open operative procedure. An incision was made on the ventral aspect of penile shaft to explore the diverticulum. Excision of the diverticulum and subsequent urethral reconstruction was performed (Figure$2,3,4)$. Postoperative recovery was uneventful.

\section{Discussion}

Congenital urethral diverticula can arise from several embryological sources. These include defects in the primordial folds and remnants of Gartner's duct. ${ }^{5}$

There are various hypothesis for the formation of the congenital urethral diverticulum. The first hypothesis is that it is a derivative of partial lacking of spongy body, representing incomplete hypospadias. ${ }^{6,7}$ Second hypothesis links it with the anterior urethral valves, appearing secondarily to these. ${ }^{7,8}$ In the third hypothesis, the congenital

1. Mohammad Afzalur Rahman

Assistant professor of Urology Department of Urology, Mymensingh Medical College, Mymensingh, Bangladesh

Address of correspondence:

Email: uroafzal@gmail.com

Mobile: +8801712080496 
urethral diverticulum arises from the spontaneous rupture of the paraurethral cysts towards the lumen of the urethra. These cysts are secondary to the obstruction of the abnormal paraurethral glands or of ectopic epithelial cell nests. ${ }^{7,8}$

Congenital urethral diverticulum may be either saccular or tubular. The saccular type has a true neck and may cause urinary obstruction since its posterior segment compresses the urethra and interrupts the urinary flow when the saccular cavity fills at the beginning of micturition. The tubular or diffuse type is located more proximally to the urethral bulb, where urinary stasis occurs along with its complications, such as urinary stasis and calculosis. $^{4}$

Congenital anterior urethral diverticulum may present itself at any age, from infant to adult. ${ }^{8}$ The symptoms depend largely on the size of the diverticulum. The smaller ones usually asymptomatic, becoming evident only when other problems such as urethritis occur. The larger diverticula tend to retain urine, harboring infection and leading to difficulties in micturition and dysuria. ${ }^{4}$ Common presenting symptom is difficulty in micturition, poor urinary stream, dribbling of urine or urinary tract infection. A careful history will reveal that the child never had a good urinary stream since birth, and a telltale sign is a cystic swelling at the penile urethra. $^{9}$ If it is uninfected and without complication, the mass is unattached to the overlying skin, nontender, and mobile laterally. On compression, urine will be seen dribbling out of the external meatus, and the swelling become empty. ${ }^{3}$

The Diagnosis of a urethral diverticulum is often clinical. Confirmation of the diagnosis is made through retrograde urethrogram (RGU) and micturating cystourethrogram (MCU). Urethrocystoscopy is also diagnostic. Ultrasonography is complimentary to the contrast studies to diagnose the condition and offers the additional advantage of evaluating the upper tracts as well.
The primary differential diagnostic conditions of anterior urethral diverticulum include anterior urethral valve (AUV), dilated Cowper's gland ducts, and post-traumatic diverticulum. The presence of a penile or penoscrotal mass clinically and the proximal lip radiologically which is seen as an arcuate filling defect should readily distinguish the diverticulum from the valve. In addition, the proximal lip forms an acute angle with the normal caliber proximal urethra in anterior urethral diverticulum, whilst in AUV, it forms an obtuse angle. ${ }^{10}$ In dilated Cowper's gland ducts, a tubular channel is seen in the ventral surface of the bulbar urethra which it parallels, and its termination is in the urogenital diaphragm. ${ }^{9}$

Management of urethral diverticulum depends on the type and size of diverticulum and the degree of obstruction. The treatment of choice for small, wellsupported diverticula is the transurethral resection (TUR) of the distal obstructing lip with a pediatric resectoscope. Whereas large diverticulum as in our case, open surgical excision and subsequent urethral reconstruction is recommended. ${ }^{3,4}$ However, the prognosis depends on the status of the upper tracts.

In summary, in patients with large anterior urethral diverticulum without any back-pressure changes, as in the present case, open diverticulectomy with primary reconstruction of urethra is recommended. This procedure carries good outcome, and it takes care of the redundant diverticular wall.

\section{Abbreviations \\ RGU: Retrograde urethrogram \\ MCU:Micturating cystourethrogram}

AUV: Anterior urethral valves

TUR: Transurethral resection 


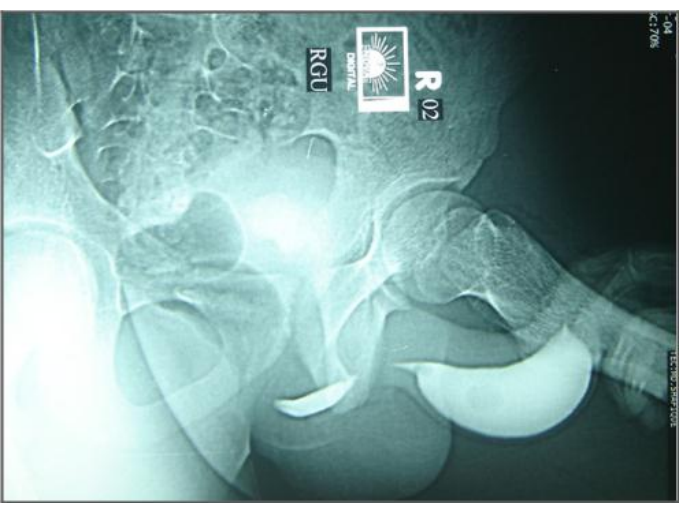

Figure 1: RGU showing large anterior diverticulum

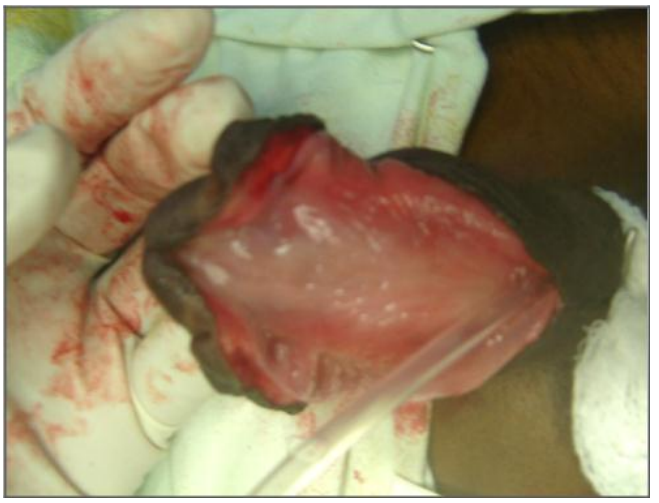

Figure 2: After exploration of diverticulum

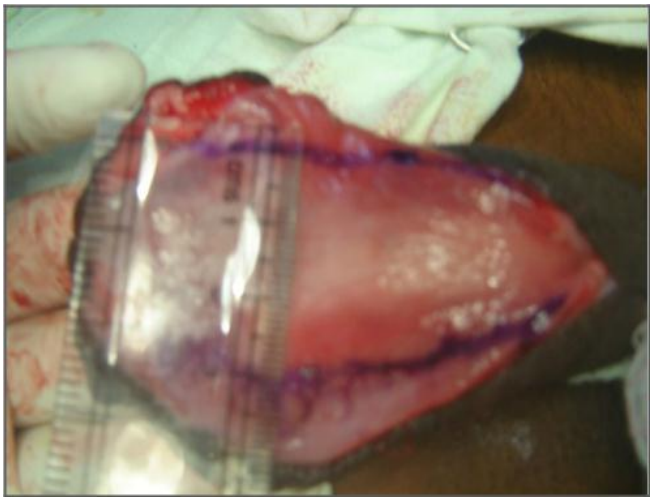

Figure 3: Planning for excision and reconstruction

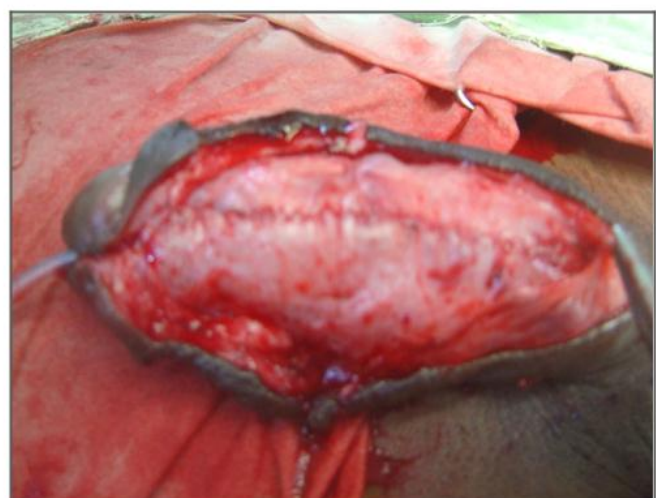

Figure 1: RGU showing large anterior diverticulum

\section{References}

1. El-Nashar, Sherif A.; Bacon, Melissa M.; KimFine, Shunaha; Weaver, Amy L.; Gebhart, John B.; Klingele, Christopher J. (2014-01-01). "Incidence of Female Urethral Diverticulum: $A$ Population-Based Analysis and Literature Review". International Urogynecology Journal. 25 (1): 73-79.

2. Hoffman, Barbara; Schorge, John; Schaffer, Joseph; Halvorson, Lisa; Bradshaw, Karen; Cunningham, F. (2012-04-12). Williams Gynecology, Second Edition. McGraw Hill Professional. ISBN 9780071716727

3. Y. S. Kadian, K. N. Rattan, Mahavir Singh, and PradeepKajal. Congenital Anterior Urethral Diverticulum in Children: A Case Report and Review. International Scholarly Research Network ISRN Surgery Volume 2011, Article ID 120307,pp:3

4. Natarajan $K$, Sivaram $P$, Thomas J, Sashidharan K. Congenital diverticulum of male urethra. Indian J Urol 2001;18:86-8.

5. Foley, Charlotte L.; Greenwell, Tamsin J.; Gardiner, Robert A. (2011-11-01). "Urethral diverticula in females". BJU International. 108 Suppl 2:20-23.

6. Dorairajan T: Defects of spongy tissue and congenital diverticula of the penile urethra. Aust NZ J sur, 32:209, 1963.

7. Sanjeet Kumar Singh, Ms Ansari. Congenital anterior urethral diverticulum, Turkish Journal of Urology, 40(3): 182-4, 2014

8. Boissonnat $P$ and Duhamel B. Congenital diverticulum the anterior urethra associated with aplasia of the abdominal muscles in a male infant. Br J Urol, 34, pp. 59-69, 1962.

9. W. Y. Cheong, H. K. Cheng, and K. P. Tan, "Congenital anterior urethral diverticulum," Singapore Medical Journal. 29(2), pp. 171-175, 1988.

10. D. K. Gupta and M. Srinivas, "Congenital anterior urethral diverticulum in children," Pediatric Surgery International. 16(8), pp. 565$568,2000$. 